\title{
Visualization and Quantification of the Microstructure Evolution of Isoprene Rubber during Uniaxial Stretching Using AFM \\ Nanomechanical Mapping
}

Xi Zhang $g^{\mathrm{b}, \mathrm{c}}$, Shuquan Sun ${ }^{\mathrm{b}, \mathrm{c}}$, Nanying Ning ${ }^{\mathrm{a}, \mathrm{b}, \mathrm{c}}$, Shouke Yan ${ }^{\mathrm{d}}$, Xiaohui $\mathrm{Wu}^{\mathrm{a}} \mathrm{b,c,c}$, , Yonglai $\mathrm{Lu}^{\mathrm{a}, \mathrm{b}, \mathrm{c}, *}$, Liqun Zhanga,b,c

a State Key Laboratory of Inorganic-Organic Composites, Beijing University of Chemical Technology, Beijing, China;

${ }^{b}$ Engineering Research Center of Elastomer Materials Energy Conservation and Resources, Ministry of Education, Beijing University of Chemical Technology, Beijing, China;

${ }^{\mathrm{c}}$ Center of Advanced Elastomer Materials, College of Material Science \& Engineering, Beijing University of Chemical Technology, Beijing, China;

d State Key Laboratory of Chemical Resource Engineering, College of Materials Science and Engineering, Beijing University of Chemical Technology, Beijing 100029, China.

*Corresponding authors:

luyonglai@mail.buct.edu.cn (Prof. Yonglai Lu)

wuxiaohui555@163.com (Dr. Xiaohui Wu) 


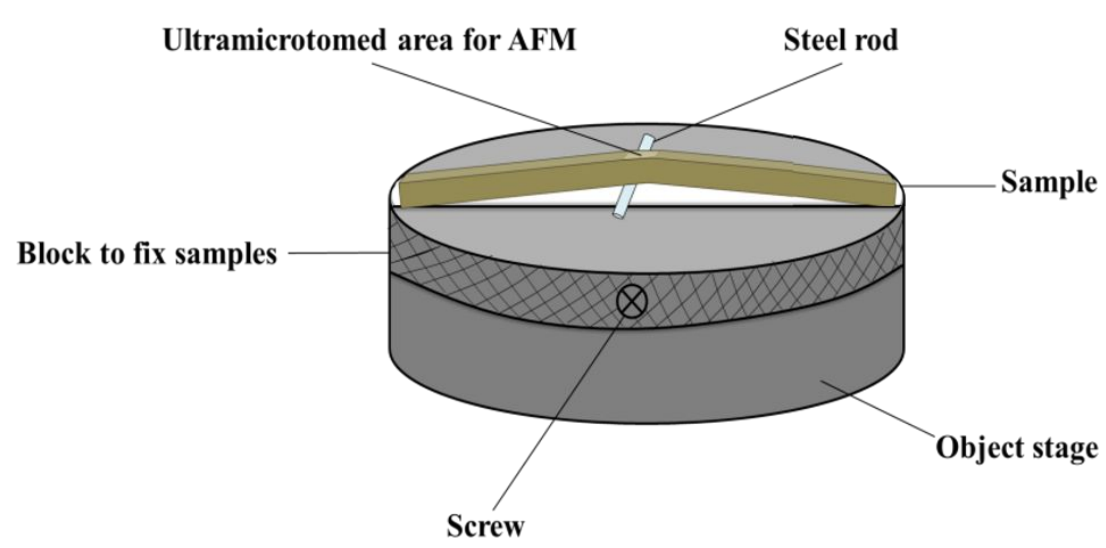

Figure S1 Sample holder for AFM measurements.
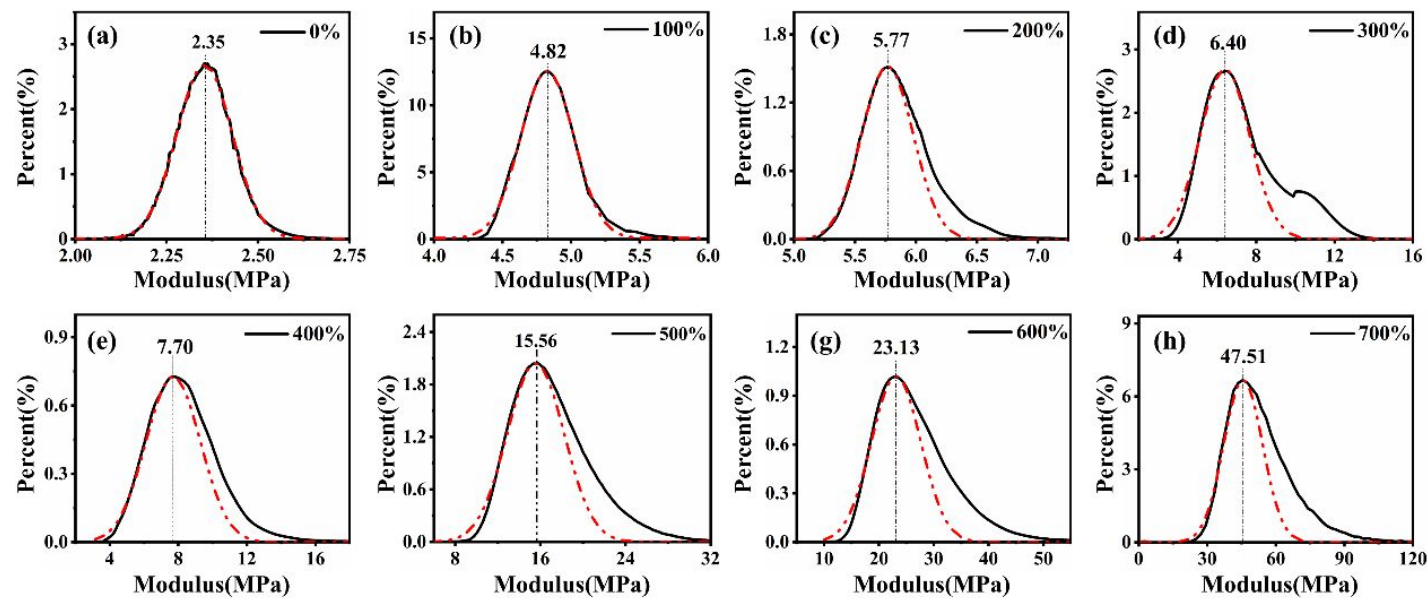

Figure S2 AFM modulus distribution profiles (black solid lines) and the one Gaussian distribution fitting results (red dotted lines) of IR at different strains: (a) 0\%, (b) 100\%, (c) $200 \%$, (d)300\%, (e) 400\%, (f) 500\%, (g) 600\%, (f) 700\%. 
S3 Asymmetry index $\delta$ calculation results:

Here, we define the asymmetry index $\delta$ to describe the heterogeneity of IR during stretching, and its calculation formula is as follows:

$$
\delta=\frac{M_{\max }-M_{0}}{M_{\min }-M_{0}}
$$

Where $M_{\max }$ is the maximum modulus of the modulus distribution profile, and $M_{\min }$ is the minimum modulus of the modulus distribution profile. $M_{0}$ is the peak modulus value of the Gaussian fitting curve in Figure S2. The asymmetry index $\delta$ of IR at different strain is calculated and shown in Figure S3.

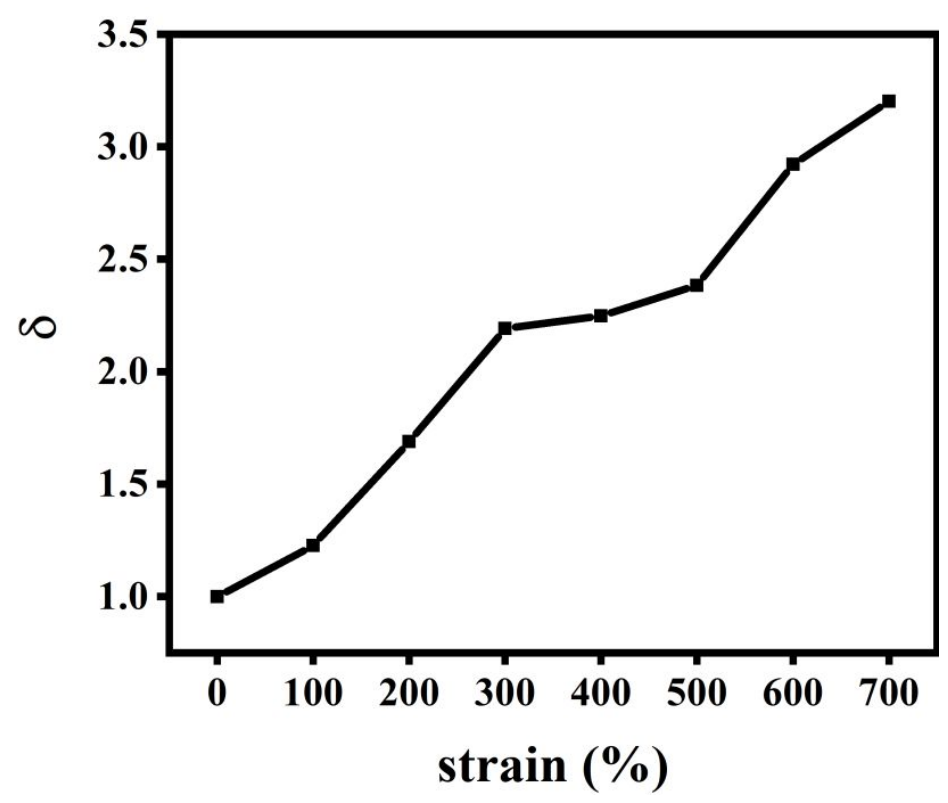

Figure S3 Asymmetry index $\delta$ of IR at different strain. 
S4 Crystallinity calculation based on the 2D-WAXD results:

2D-WAXD patterns are integrated into the one-dimensional profiles. Then, onedimensional integrated intensity profiles are deconvoluted into several Gaussian peaks and crystallinities are determined by taking the ratio of integrated areas of the crystal peaks to the total integrated area.
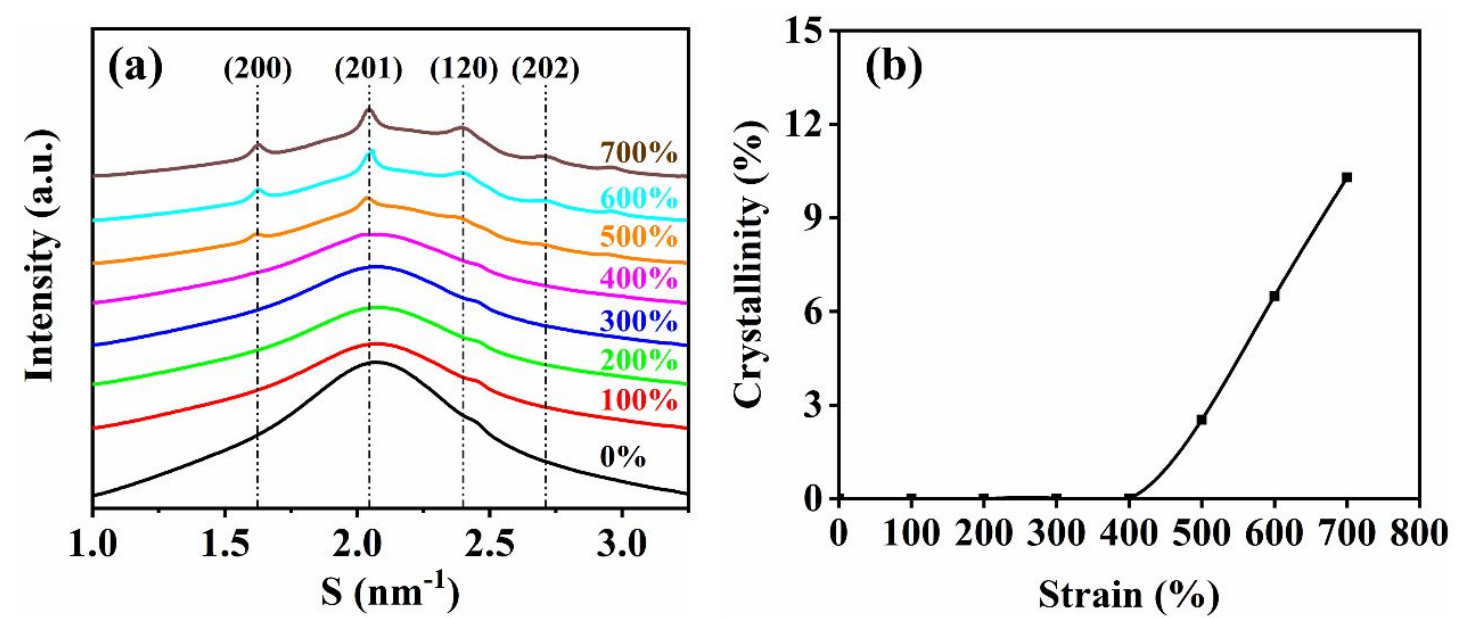

Figure S4 (a) One-dimensional WAXD profiles and (b) crystallinity at different strains of IR sample. 